TAMKANG JOURNAL OF MATHEMATICS

Volume 22, Number 4, Winter 1991

\title{
A NOTE ON SIMPLE EXTENSIONS AND SEMI-COMPACT TOPOLOGIES
}

\author{
MAXIMILIAN GANSTER
}

\begin{abstract}
We study simple extensions of semi-compact topological spaces. Our main result says that if $X$ is an infinite set then maximal semi-compact topologies
on $X$ do not exist.
\end{abstract}

\section{Introduction and Preliminaries}

Let $S$ be a subset of a topological space $(X, \tau)$. The closure and the interior of $S$ with respect to $(X, \tau)$ will be denoted by $c l_{\tau} S$ and int $S$ respectively. If $S \subset c l_{\tau}\left(\right.$ int $_{\tau} S$ ) then $S$ is called semi-open [3] in $(X, \tau)$, and if $\operatorname{int}_{\tau}\left(c l_{\tau} S\right)=\phi$ then $S$ is said to be nwd (= nowhere dense) in $(X, \tau)$. The set of natural numbers will be denoted by $\omega$.

Let $A$ be a subset of a space $(X, \tau)$. The simple extension of $(X, \tau)$ by $A[4]$ is the space $(X, \sigma)$ where $\sigma=\{U \cup(V \cap A): U, V \in \tau\}$. It is pointed out in [4] that for any subset $B$ of $X$ we have $c l_{\sigma} B=c l_{\tau} B \cap\left((X-A) \cup\left(A \cap c l_{\tau}(B \cap A)\right)\right)$. Consequently, $c l_{\tau} B \cap(X-A) \subset c l_{\sigma} B$ for any $B \subset X$, and $c l_{\tau} B=c l_{\sigma} B$ whenever $B \subset A$. From these observations one can infer immediately the following result.

Lemma 1.1 Let $(X, \sigma)$ be the simple extension of $(X, \tau)$ by $A \subset X$, and let $N \subset X$ be nwd in $(X, \sigma)$.

i) If $A$ is closed and nwd in $(X, \tau)$ then $N$ is nwd in $(X, \tau)$.

ii) If $N \subset A$ then $N$ is nwd in $(X, \tau)$.

\section{Semi-Compact Expansions}

A space $(X, \tau)$ is called semi-compact [2] if every cover of $X$ by semi-open subsets has a finite subcover. Recall that $(X, \tau)$ is said to be semi-irreducible [5] if every disjoint family of nonempty open sets is finite. We will make use of the following characterization of semi-compactness which is due to Dorsett [2].

Theorem 2.1. A space $(X, \tau)$ is semi-compact if and only if $(X, \tau)$ is semiirreducible and every nwd subset of $(X, \tau)$ is finite.

Receive November 30, 1990. 
Our next result about the preservation of semi-irreducibility will also be needed in the sequel.

Lemma 2.2 Let $(X, \tau)$ be semi-irreducible and let $(X, \sigma)$ be the simple extension of $(X, \tau)$ by $A \subset X$. If $A$ is either dense in $(X, \tau)$ or finite then $(X, \sigma)$ is semi-irreducibile.

Proof. Suppose that $\left\{W_{n}: n \in \omega\right\}$ is a disjoint family of nonempty open sets in $(X, \sigma)$. Let $W_{n}=U_{n} \cup\left(V_{n} \cap A\right)$ with $U_{n}, V_{n} \in \tau$ for each $n \in \omega$. Since $(X, \tau)$ is semi-irreducibile there exists $k \in \omega$ such that $U_{n}$ is empty whenver $n \geq k$. Hence $\left\{V_{n} \cap A: n_{\geq} k\right\}$ is a disjoint family of nonempty sets which is impossible in the case that $A$ is finite. If $A$ is dense in $(X, \tau)$ then $V_{n} \cap V_{m} \cap A=\phi$ for $n, m \geq k$, and so $\left\{V_{n}: n \geq k\right\}$ is a disjoint family of nomempty open sets in $(X, \tau)$, a contradiction.

By using the facts we have carried together so far we are now able to show that certain simple extensions are semi-compact.

Theorem 2.3. Let $(X, \tau)$ be semi-compact and suppose that $\left\{x_{0}\right\}$ is not open for some $x_{0} \in X$. If $(X, \sigma)$ denotes the simple extension of $(X, \tau)$ by $A=X-\left\{x_{0}\right\}$ then $(X, \sigma)$ is semi-compact.

Proof. Since $A$ is dense in $(X, \tau),(X, \sigma)$ is semi-irreducibile by Lemma 2.2. Let $N \subset X$ be nwd in $(X, \sigma)$. By Lemma 1.1 ii) $N \cap A$ is $n w d$ in $(X, \tau)$ and hence finite. Consequently, $N$ is finite and $(X, \sigma)$ is semi-compact by Theorem 2.1 .

Theorem 2.4. Let $(X, \tau)$ be semi-compact and suppose that $\left\{x_{0}\right\}$ is closed but not open for some $\mathfrak{x}_{0} \in X$. If $(x, \sigma)$ denotes the simple extension of $(X, \tau)$ by $A=\left\{x_{0}\right\}$ then $(X, \sigma)$ is semi-compact.

Proof. $(X, \sigma)$ is semi-irreducibile by Lemma 2.2. Let $N \subset X$ be nwd in $(X, \sigma)$. Since $A$ is closed and nwd in $(X, \tau), N$ is nwd in $(X, \tau)$ by Lemma 1.1 i). Hence $N$ is finite and $(X, \sigma)$ is semi-compact by Theorem 2.1 .

\section{Maximal Semi-Compact Spaces}

If $X$ is a finite set and if $\tau$ denotes the discrete topology on $X$ then $(X, \tau)$ is obviously maximal semi-compact. It turns out that this is the only possibility for a space to be maximal semi-compact.

Theorem 3.1. There are no maximal semi-compact topologies on an infinite set.

Proof. Suppose that $(X, \tau)$ is maximal semi-compact and $X$ is infinite. Then $(X, \tau)$ is clearly not the discrete space and so there exists $x_{0} \in X$ such that $\left\{x_{0}\right\}$ is not open in $(X, \tau)$. Since $(X, \tau)$ is maximal semi-compact it follows from Theorem 2.3 that $\left\{x_{0}\right\}$ is closed in $(X, \tau)$. But now Theorem 2.4 implies that the simple extension of $(X, \tau)$ by 
$\left\{x_{0}\right\}$ is semi-compact. This obviously produces a contradiction since $\left\{x_{0}\right\}$ is not open in $(X, \tau)$.

Remark 3.2. In [1] several results about maximal semi-compact spaces are presented. These results, based on Lemma 2.1 in [1], seem to be contradictory to our results. It should be noted, however, that Lemma 2.1 in [1] is slightly incorrect and a possible correct version would be: "If $R$ and $\mathbb{R}^{\prime}$ are two properties of a space $(X, \tau)$ such that $\mathbb{R}$ implies $\mathbb{R}^{\prime}$, then $(X, \tau)$ is maximal $\mathbb{R}$ if it is $R$ and maximal $\mathbb{R}^{\prime \prime}$.

\section{References}

[1] M. E. Abd El-Monsef and A. M. Kozae, "Maximal S-closed and maximal semi-compact topologies", Tamkang J. Math. 20 (4) (1989), pp. 283-288.

[2] C. Dorsett, "Semi-compactness, semi-separation axioms and product spaces", Bulll. Malaysian Math. Soc. 4 (2) (1981), pp. 21-28.

[3] N. Levine, "Semi-open sets and semicontinuity in topological spaces", Amer. Math. Monthly 70 (1963), pp. 36-41.

[4] N. Levine, "Simple extensions of topologies", Amer. Math. Monthly 71 (1964), pp. 22-25.

[5] A. H. Stone, "Hereditarily compact spaces", Amer. J. Math. 82 (1960), pp. 900-916.

Department of Mathematics, Graz University of Technology, Steyrergasse 30, A-8010 Graz; Austria. 


\title{
ON SOME INTEGRAL OPIRATORS FOR CIRTAIN FAMTLIES OF ANALYTIC FUNCTIONS
}

\author{
KHALIDA INAYAT NOOR
}

\begin{abstract}
In this paper, some classes of analy tic functions related with functions of bounded boundary rotation are defined and discussed with reference to certain integral operators.
\end{abstract}

Keywords and Phrases: Subordinate, bounded boundary rotation, starlike, convex, closeto-convex, bounded radius rotation.

\section{Intróduction}

Let $f$ be analytic in $E=\{z:|z|<1\}$, and given by

$$
f(z)=z+\sum_{n=2}^{\infty} a_{n} z^{n} .
$$

A function $g$, analytic in $E$, is called subordinate to a function $G$ if there exists a Schwarz function $w(z)$ analytic in $E$ with $w(0)=0$ and $|w(z)|<1$ in $E$ such that
$g(z)=G(w(z))$.

In [1], Janowski introduced the class $P[A, B]$. For $A$ and $B,-1 \leq B<A \leq 1$, a function $p$, analytic in $E$ with $p(0)=1$ belongs to the class $P[A, B]$ if $p(z)$ is subordinate to $\frac{1+A z}{1+B z}$. When $A=1, B=-1, P[1,-1] \equiv P$, the class of analytic functions with positive real part. We generalize this concept to define the class $P_{k}[A, B], k \geq 2$. A function $p \in P_{k}[A, B]$ if, and only if, there exist $p_{1}, p_{2} \in P[A, B]$ such that

$$
p(z)=\left(\frac{k}{4}+\frac{1}{2}\right) p_{1}(z)-\left(\frac{k}{4}-\frac{1}{2}\right) p_{2}(z)
$$

Definition 1.1. A function $f$, analytic in $E$, and given by (1.1) is said to belong to the class $R_{k}[A, B], k \geq 2$, if and only if, $\frac{z f^{\prime}(z)}{f(z)} \in P_{k}[A, B]$.

Receive January 7, 1991.

1980 AMS(MOS) Suject Classfication: 30A32, 30A34, 30C45 
For $A=1, B=-1$, we obtain $R_{k}[1,-1] \equiv R_{k}$, the class of bounded radius rotation discussed in [2], and $R_{2}[1,-1] \equiv S^{*}$, the class of starlike univalent functions. Also $R_{2}[A, B] \equiv S^{*}[A, B] \subset S^{*}$, see $[3]$.

Similarly we have:

Definition 1.2. Let $f$ be analytic in $E$ and given by (1.1). Then $f \in V_{k}[A, B]$, $k \geq 2$, if and only if $\frac{\left(z f^{\prime}(z)\right)}{f^{\prime}(z)} \in P_{k}[A, B]$.

If $A=A, B=-1$, then $V_{k}[1,-1] \equiv C$, the class of convex univalent functions.

Also $V_{2}[A, B] \equiv C[A, B] \subset C$, see [3]. It is clear that

$$
f \in V_{k}[A, B] \Longleftrightarrow z f^{\prime} \in \mathbb{R}_{k}[A, B]
$$

Definition 1.3. Let $f$ be analytic in $E$ and given by (1.1). Then $f$ is said to belong to the class $T_{k}[A, B], k \geq 2$, if and only if, there exists a function $g \in V_{k}[A, B]$ such that $\frac{f^{\prime}(z)}{g^{\prime}(z)} \in P[A, B]$.

We note that:

i. $T_{2}[1,-1] \equiv K$, the class of close-to-convex functions introduced and studied by Kaplan [4].

ii. $T_{k}[1,-1] \equiv T_{k}$, a class of analytic functions introduced and studied in [5].

iii. $T_{2}[A, B] \equiv \mathbb{K}[A, B]$, which has been studied in a more general way in [3].

\section{Preliminary Results}

Lemma 2.1. Let $p \in \mathbb{P}_{k}[A, B]$. Then $p \in P$ for $|z|<r_{1}$, where

$$
r_{1}=4 /\left[k(A-B)+\sqrt{k^{2}(B-A)^{2}+16 A B}\right] .
$$

This result is sharp.

Proof. Now $p \in P_{k}[A, B]$ implies that

$$
p(z)=\left(\frac{k}{4}+\frac{1}{2}\right) p_{1}(z)-\left(\frac{k}{4}-\frac{1}{2}\right) p_{2}(z), p_{1}, p_{2} \in P[A, B] .
$$

Since

we have

$$
\frac{1-A r}{1-B r} \leq \operatorname{Rep}_{i}(z) \leq\left|P_{i}(z)\right| \leq \frac{1+A r}{1+B r}, i=1,2, \text { see }[6]
$$

$$
\begin{aligned}
\operatorname{Re} p(z) & \geq\left(\frac{k}{4}+\frac{1}{2}\right) \frac{1-A r}{1-B r}-\left(\frac{k}{4}-\frac{1}{2}\right) \frac{1+A r}{1+B r} \\
& =\frac{1+\frac{k}{2}(B-A) r-A B r^{2}}{1-B^{2} r^{2}} .
\end{aligned}
$$


From the above inequality, it is clear that $\operatorname{Re} p(z) \geq 0$ for $|z|<r_{1}$, where $r_{1}$ is given by (2.1).

The sharpness follows from the function $p_{0}$, where

$$
p_{0}(z)=\frac{1}{2}\left[\left(\frac{k}{2}+1\right) \frac{1-A z}{1-B z}-\left(\frac{k}{2}-1\right) \frac{1+A z}{1+B r}\right] .
$$

We shall need the following extension of Libera's Lemma [7].

Lemma 2.2. [6]: Let $N$ and $D$ be analytic in $E, D$ map $E$ noto a manysheeted starlike region. $N(0)=0=D(0), N^{\prime}(0)=1=D^{\prime}(0)$, and

Then

$$
\frac{N^{\prime}(z)}{D^{\prime}} \in P[A, B]
$$

$$
\frac{N(z)}{D(z)} \in P[A, B] \text {. }
$$

Lemma 2.3. [8]: Let $p_{1}$ and $p_{2} \in P[A, B]$. Then for $\alpha, \beta$ any positive reals

$$
\frac{1}{\alpha+\beta}\left[\alpha p_{1}(z)+\beta p_{2}(z)\right] \in p[A, B] \text {. }
$$

3. Main Results

Theorem 3.1. Let $f \in V_{k}[A, B]$ and $g \in R_{k}[A, B]$. Let $H$ be defined by

$$
H(z)=\int_{0}^{z}\left(f^{\prime}(t)\right)^{\alpha}\left(\frac{g(t)}{t}\right)^{\beta} d t
$$

where $\alpha$ and $\beta$ are positive reals with $\alpha+\beta=1$. Then $H \in V_{k}[A, B]$.

Proof. From (3.1), we have

$$
H^{\prime}(z)=\left(f^{\prime}(z)\right)^{\alpha}\left(\frac{g(z)}{z}\right)^{\beta} .
$$

Logarithemic differentiation yields

$$
\begin{aligned}
\frac{\left(z H^{\prime}(z)\right)^{\prime}}{H^{\prime}(z)} & =\alpha \frac{\left(z f^{\prime}(z)\right)^{\prime}}{f^{\prime}(z)}+\beta \frac{z g^{\prime}(z)}{g(z)} \\
& =\alpha p_{1}(z)+\beta p_{2}(z), p_{1}, p_{2} \in P_{k}[A, B] \\
& =\alpha\left[\left(\frac{k}{4}+\frac{1}{2}\right) q_{1}(z)-\left(\frac{k}{4}-\frac{1}{2}\right) q_{2}(z)\right]+\beta\left[\left(\frac{k}{4}+\frac{1}{2}\right) h_{1}(z)-\left(\frac{k}{4}-\frac{1}{2}\right) h_{2}(z)\right],
\end{aligned}
$$


where $q_{i}, h_{i} \in P[A, B], i=1,2$.

Thus, using Lemma 2.3, we have

$$
\frac{\left(z H^{\prime}(z)\right)^{\prime}}{H^{\prime}(z)}=\left(\frac{k}{4}+\frac{1}{2}\right) q(z)-\left(\frac{k}{4}-\frac{1}{2}\right) h(z),
$$

$q, h \in P[A, B]$ and this gives us the required result that $H \in V_{k}[A, B]$.

Following the same technique used in Theorem 3.1, we have: Then

Theorem 3.2. Let $f_{i} \in V_{k}[A, B], i=1,2, \ldots, n$.

$$
K(z)=\int_{0}^{z} \pi_{i}\left(f_{1}^{\prime}(t)\right)^{\alpha_{i}} d t, \quad \sum_{i=1}^{n} \alpha_{i}=1
$$

belongs to $V_{k}[A, B]$.

Theorem 3.3. Let $f \in R_{k}[A . B]$ and let

$$
F(z)=\left[(\alpha+\nu+\eta) z^{-\nu} \int_{0}^{z}(f(t))^{\alpha} t^{\nu+\eta-1} d t\right]^{\frac{\lambda}{\alpha+\eta}}
$$

where $\alpha>0, \eta \geq 0$ and $\operatorname{Re}(\nu+\eta) \geq 0$. Then $F$ maps $|z|<r_{1}$ onto a star-shaped domain, $r_{1}$ is given by (2.1).

In (3.2) all powers are principal ones.

Proof. Differentiating (3.2) logarithemically, we obtain

$$
\frac{z F^{\prime}(z)}{F(z)}=\frac{f^{\alpha}(z) z^{\nu+\eta}-\nu \int_{0}^{z} f^{\alpha}(t) t^{\nu+\eta-1} d t}{(\alpha+\eta) \int_{0}^{z} f^{\alpha}(t) t^{\nu+\eta-1} d t}=\frac{N(z)}{D(z)}
$$

We note that $N(0)=0=D(0)$, and by a Lemma due to Bermardi [9], $D(z)$ is $(\nu+\eta+\alpha-1)$ - valent starlike for $|z|<r_{1}$, where $r_{1}$ is given by (2.1). Also

that is

$$
\frac{N^{\prime}(z)}{D^{\prime}(z)}=\frac{\alpha z f^{\prime}(z)}{(\alpha+\eta) f(z)}+\frac{\eta}{\alpha+\eta}
$$

$$
\frac{N^{\prime}(z)}{D^{\prime}(z)} \in P\left[1-\frac{2 \eta}{\alpha+\eta},-1\right]
$$

for all $|z|<r$. Using lemma 2.2 with $A=1-\frac{2 \eta}{\alpha+\eta}, B=-1$, we see that $\frac{N(z)}{D(z)} \in$ $P\left[1-\frac{2 \eta}{\alpha+\eta}-1\right]$ for $|z|<r_{1}$. Hence $F \in S^{*}\left[1-\frac{2 \eta}{\alpha+\eta},-1\right] \subset S^{*}$ for $|z|<r$, and this gives us the required reult.

From the relationship (1.3) and the fact that $f$ is convex if and only if $z f^{\prime}$ is starlike, we immediately have the following: 
Theorem 3.4. Let $f \in V_{k}[A, B]$. Then $F$, defined by (3.2), maps $|z|<r_{1}$ onto a convex domain, where $r_{1}$ is given by (2.1).

Theorem 3.5. Let $f \in T_{k}[A, B]$ with respect to $h \in V_{k}[A, B]$. Let $g \in R_{k}[A, B]$, and for $\alpha+\beta=1, \alpha, \beta \geq 0$, let $F$ be defined as

$$
F(z)=\int_{0}^{z}\left(f^{\prime}(t)\right)^{\alpha}\left(\frac{g(t)}{t}\right)^{\beta} d t
$$

Then $F$ is close-to-convex with respect to $H$ defined by

$$
H(z)=\int_{0}^{z}\left(h^{\prime}(t)\right)^{\alpha}\left(\frac{g(t)}{t}\right)^{\beta} d t
$$

for all $|z|<r, r_{1}$ given by (2.1).

Proof. From Theorem 3.1, we see that $H \in V_{k}[A, B]$.

Now

$$
\begin{aligned}
\frac{F^{\prime}(z)}{H^{\prime}(z)} & =\left(f^{\prime}(z)\right)^{\alpha}\left(\frac{g(z)}{t}\right)^{\beta} /\left(h^{\prime}(z)\right)^{\alpha}\left(\frac{g(z)}{z}\right)^{\beta} \\
& =\left(\frac{f^{\prime}(z)}{h^{\prime}(z)}\right)^{\alpha}=p_{1}^{\alpha}(z) \in P[A, B], 0 \leq \alpha \leq 1 .
\end{aligned}
$$

Hence the results.

\section{References}

1. W. Janowski, "Some extremal problems for certain families of analytic functions $I$ ", Ann. Polon. Math. 28 (1973), pp. 297-326.

2. V. Karuna Karan and K. Padma, "Functions of bounded radius rotation", Indian J. Pure appl. Math., 12 (1981), pp. 621-627.

3. E. M. Silvia, "Subclasses of close-to-convex functions", Int. J. Math. \& Math. Sci. 6 (1983), pp. 449-458.

4. W. Kaplan, "Close-ta-convex Schlicht functions", Mich. Math. J. 1 (1952), pp. 169-185.

5. K. I. Noor, "On a generalization of close-to-convexity", Int. J. Math. \& Math. Sci. 6 (1983), pp.327334.

6. R. Parvatham and T. N. Shanmugam, "On analytic functions with reference to an integral operator", Bull. Austral. Math. Soc. 28 (1983), pp. 207-215.

7. R. J. Libera, "Some classes of regular univalent functions", Proc Amer. Match. Soc. 16 (1965), pp. 755-758.

8. K. I. Noor, "On Some Univalent Integral Operators", J. Math. Anal. Appl. 128 (1987), pp. 586-592.

9. S. D. Bernardi, "Convex and starlike univalout functions", Trans. Amer. Math. Soc. 135 (1969), pp. 429-446.

Mathematics Department, College of Science, P. O. Box 2455, King Saud University, Riyadh 11451, Saudi Arabia. 